\title{
An Integrated Geometric Modeling Methodology for 2.5D Cylindrical Prismatic Part for Computer Aided Process Planning
}

\author{
Viswa Mohan Pedagopu', Manish Kumar² \\ ${ }^{1}$ Department of Mechanical Engineering, Shoolini University, Solan, Bajhol, India \\ ${ }^{2}$ JNV University, Jodhpur, India \\ Email: viswamohanpedagopu@rocketmail.com
}

Received 13 September 2014; revised 5 October 2014; accepted 16 October 2014

Copyright (C) 2014 by authors and Scientific Research Publishing Inc.

This work is licensed under the Creative Commons Attribution International License (CC BY). http://creativecommons.org/licenses/by/4.0/

cC) (7) Open Access

\section{Abstract}

The field of solid modeling has created numerous techniques for unambiguous computer representations of three-dimensional objects. Its data structures and algorithms have been used in a broad range of applications: Computer-Aided Design and Computer-Aided Manufacturing (CAD/ CAM), robotics, computer vision, computer graphics and visualization, virtual reality, etc. This research paper is used to generate process plan from feature-based modeling, based on an integrated geometric modeling system that supports both feature-based modeling and information storage. Present system is developed only for milling components and limited to selective machining features for prismatic components and further implemented for more machining features to develop algorithms for modeling the components through the input of machining features. As a result, feature information is directly available to downstream activities, and feature extraction is no longer needed. The various systematic steps involved in this approach are study of Design, identification of Features, selection of Processes, Tools and Machines, Machining and Inspection [DFPTMMI]. Machining features generated in the design stage are recognized and stored under the Visual Basic control of CATIA software ActiveX interface. Algorithms are developed for individual features and these algorithms are embedded in Visual Basic forms. This system is discussed and suited for 2.5 Dimensional part approach, however, that can be extended to 3 dimensional prismatic part and complex features machining. Finally a process planning chart has been presented as a model process planning.

\section{Keywords}

Modeling, Feature, Computer Aided Manufacturing, Design, Prismatic Part

How to cite this paper: Pedagopu, V.M. and Kumar, M. (2014) An Integrated Geometric Modeling Methodology for 2.5D Cylindrical Prismatic Part for Computer Aided Process Planning. Intelligent Control and Automation, 5, $183-189$. 


\section{Introduction}

CAPP is usually considered to be part of Computer-Aided Manufacturing (CAM) [1]. However, this tends to imply that CAM is a stand-alone system. In fact, a synergy results when CAM is combined with computer-aided design to create a CAD/CAM system [2]. In such a system, CAPP becomes the direct connection between design and manufacturing [3]. Process planning includes identification of the processes, machine tools, cutting tools, setups and fixtures to produce the desired product, along with geometric information [4]. Even today, the activities of process planning are partially based on the skill of experienced process planners, which results in time-consuming procedures [5]. As process planning is very complex, it would be desirable to use computeraided approaches to relieve the process planner from routine activities and reduce the time and cost of the task [6]. Because of the need to respond quickly to highly variable market demands, the development of computeraided process planning (CAPP) systems is necessary. To achieve this computer integrated manufacturing (CIM) system, information about machining component is must. In terms of integrating CAPP systems [7], the feature-based approaches have been recognized as essential tools for eventually integrating process planning and design [8]. Feature-based approaches are divided into two groups, namely, feature recognition and design by feature [9]. The feature recognition approach examines the topology and geometry of a part and matches them with the appropriate definition of predefined features [10].

The researchers have found that the feature recognition processor first translates the design feature model of a part into an intermediate manufacturing feature tree by handling design features and then final manufacturing tree is updated with some interpretations [11]. They proposed algorithms based on progressive Z-maps for recognizing the machining features and feature topologies by analyzing NC programs [12]. Researchers presented a new approach to extracting machining features from a feature-based design model, which supports both feature-based modeling and feature recognition [13]. Feature recognition is achieved through an incremental feature converter [14]. The scientist has given a novel feature finder, which automatically generates a part interpretation in terms of machining features, by utilizing information from a variety of sources such as nominal geometry, tolerances and attributes, and design features [15]. They presented system architecture for feature-based modeling which is founded on integration that is obtained through the definition of a common feature library and an intermediate model, which plays the role of communication link between the geometric model and the feature-based model [16].

\section{Process Planning}

The preparation and execution of a manufacture of products carried out by process planning including the selection, defining a process to get optimum result. The actual transformation of rugged raw structure to end user is not as simply as say, obviously involves various complex approaches includes, the study and understanding of product model design. According to which bifurcation of features, selection of related machine tools milling, turning and drilling etc., determination of number of set ups, fixture design, methods of machining and sequences, tool path trajectory, cutting tool control conditions, preparation of CNC programming ,capacity and inspection planning. The process planning is much more important particularly in the job-shop type of manufacturing industries (See Figure 1).

The production facilities are subjected to relatively small changes over time, which includes processes, machine tools, cutting tools, and measuring machines etc. are not mostly subjected to great changes over time. Therefore, designers must take into account these manufacturing constraints. The main of promoting the Computer Aided Process Planning (CAPP) in mass production, large numbers of small batches put a great burden on process planning departments where skilled workforce is scarce. As diverse process planners make dissimilar process plans for the product of same geometrical structure, many companies have different process plans for the same part, resulting in inconsistencies and extra paper work CAPP systems which lead wastage of time, process and cost. However, the CAPP system reduces the demand on the skilled planner, the process planning, and manufacturing cost, creates consistent plans, produces accurate plans and increases productivity.

Now, more advanced CAPP systems take a CAD based product model as input. At best, this is a 3D solid model on which the CAPP system can perform automatic feature recognition. However, some CAPP systems exist that take 2.5D models as an input and on which the process planner has to identify the manufacturing features either manually or automatically. So many researchers worked on the process planning contain tolerance and material information. Some CAPP systems allow for adding this information to the product model manually in order to allow automatic reasoning. 


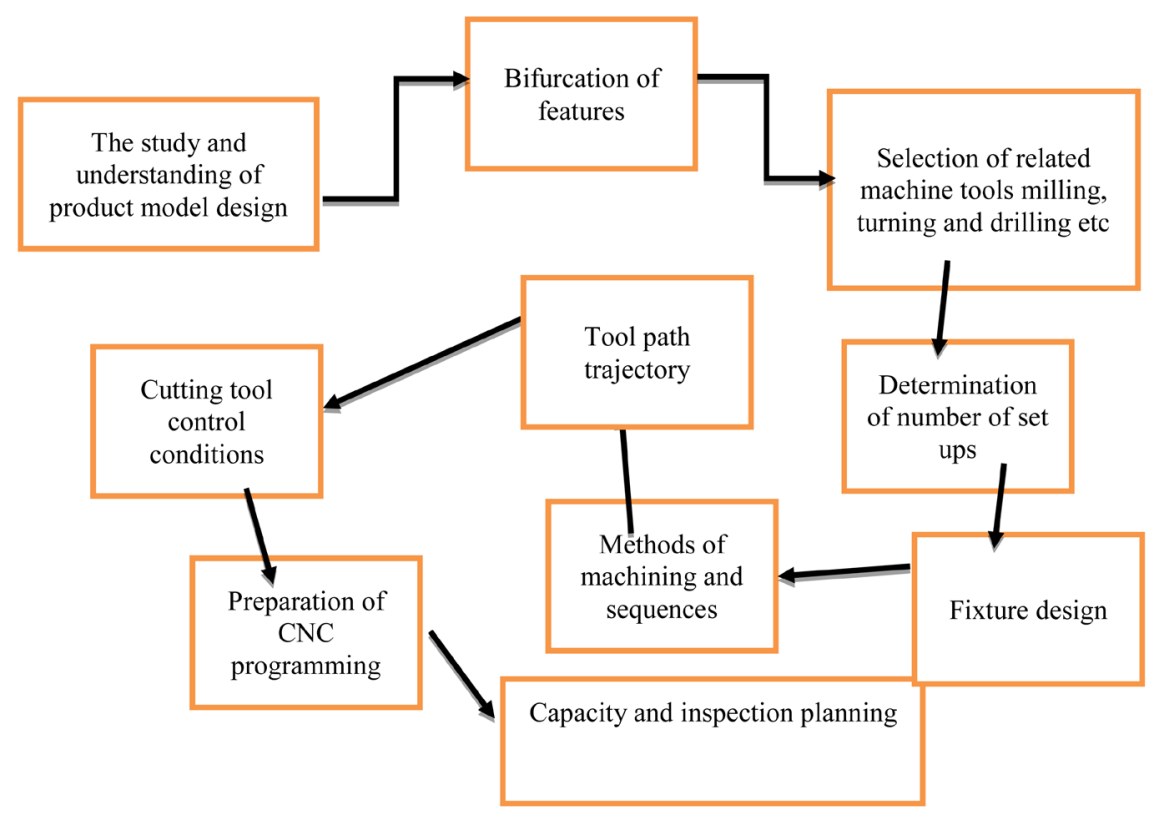

Figure 1. The flow chart for process planning.

\section{Feature-Based Modeling Technique a Proposed Approach}

In this paper, new approach to include machining features from a feature-based solid modeling, based on an integrated geometric modeling system that supports both feature-based modeling and feature recognition is proposed. Three modules are integrated viz. design module, information storage module and process plan generation model. Design module consists of feature based modeling system, which is developed using visual basic in integration with CATIA software for milling components. This feature-based solid modeling based on an integrated geometric modeling system that supports both feature-based modeling and feature identification. Information storage module stores the information available in the design stage and connects both design module and process plan module. The information of the machining features available in storage module is used in process plan generation of the components. The proposed feature based modeling system provides a graphical environment for solid modeling with the help of catia software. In the proposed system, design module provides inbuilt features such as plane cylinder, taper cylinder, through hole, blind hole and groove to generate the model. Algorithms are developed for individual features and these algorithms are embedded in Visual Basic forms. Required dimensions of the selected feature are specified by the user in the user interactive mode. The algorithm for modeling and cylindrical prismatic part based on the dimensions entered by the manipulator is represented below.

The model created and is shown in Figure 2.

1. In the ZX plane the length and radius of cylindrical prism is well-thought-out and stored in the DATA base. and the identical is recollected when and where is required. The $\mathrm{Z}$ axis is always in negative value.

2. For the first feature machining the referenced point always be ZX plane and then for the next feature the coordinates of the center point of the previous machining feature is considered as starting point for the present one.

3. The starting point of the feature is computed from the origin of the model, if the previous history is null then start from reference point.

4. Knots indicating the starting point of the feature, radius, and length of the feature machining are created in the information storage module.

5. Dimensions of the machining feature like length, radius, and reference point are stored in database.

The model then fragmented in accordance with machine features as shown in Figure 3.

\subsection{Algorithm for through Hole}

The following algorithm gives information about modeling and storing the feature data of a through hole. The model created and feature list stored is shown in Figure 4. 


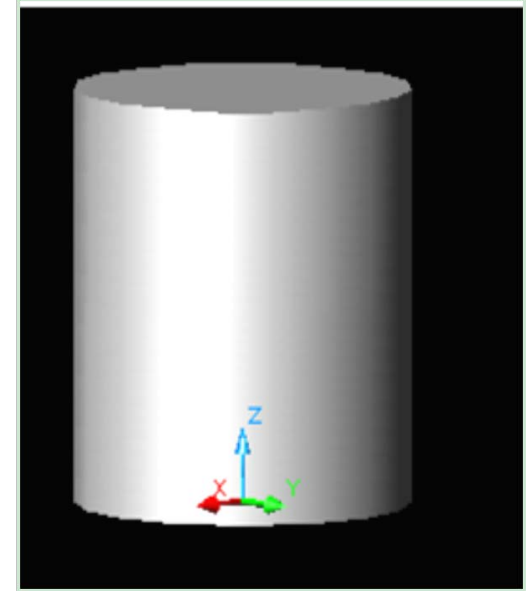

Figure 2. Cylindrical prismatic component.

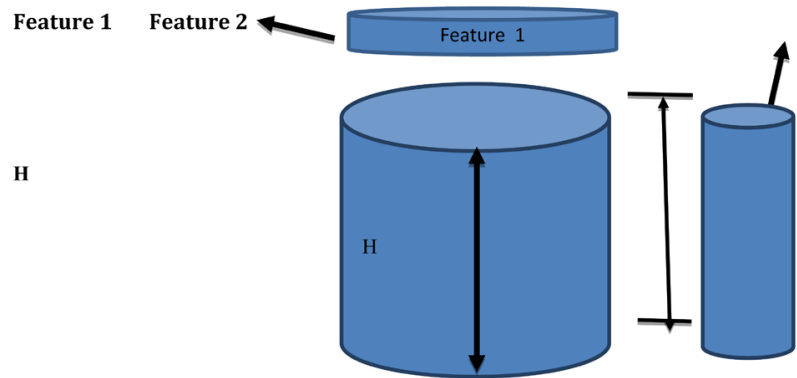

Figure 3. Extraction of features for machining.

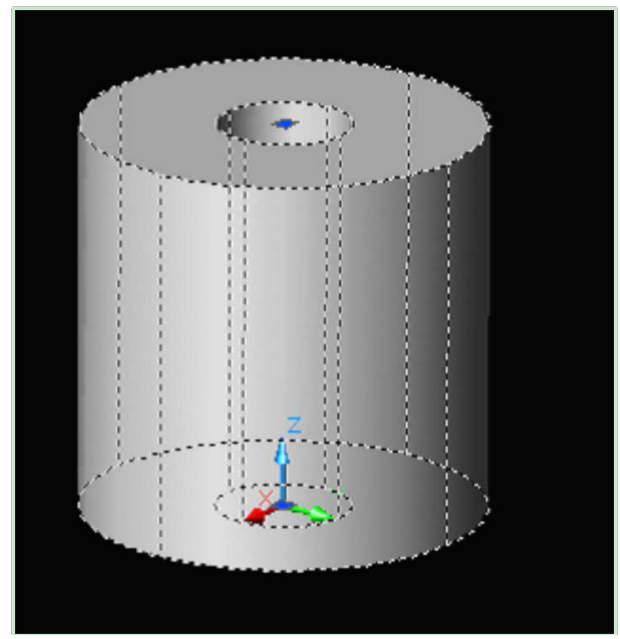

Figure 4. Prismatic part of cylindrical final shape.

1. The depth of the hole from the first end point to second end point of the hole is specified and distance between these two points should be always greater than the total length of the component due to through hole.

2. Radius of the hole is quantified and the Hole is specified with dimensions and the feature information radius and length of the hole.

\subsection{Algorithm for Blind Hole}

The algorithm for a blind hole is explained with the help of Figure 5(a) and Figure 5(b). 


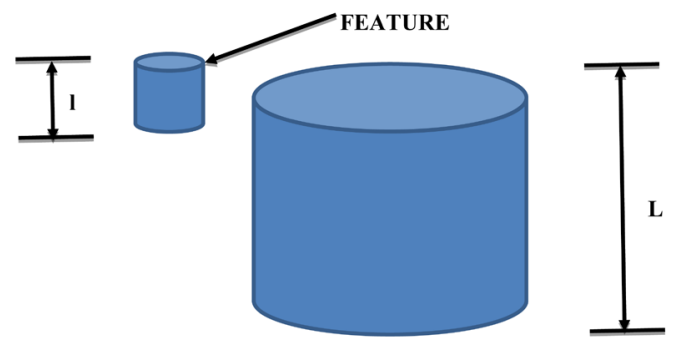

(a)

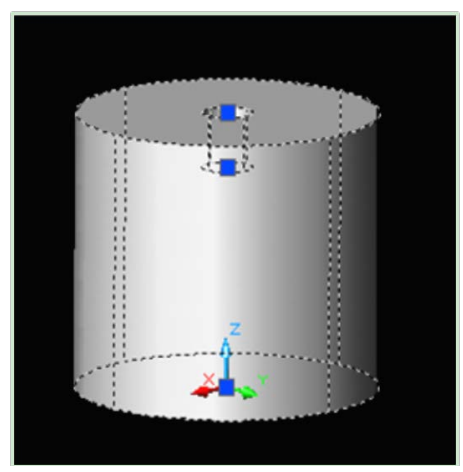

(b)

Figure 5. Blind hole with its feature list.

1. Starting point and ending point of the hole is specified and distance between these two points should be always less than the total length or height of the component and the depth of the drill is equal to the length of the hole.

2. Radius of the hole is specified.

Blind hole is created with specified dimensions and the feature information radius and length of the hole are stored and displayed in the feature list window using nodes or knots.

\section{Process Plan Generation}

Existing CAD systems commonly represent geometric models in terms of elementary geometric entities such as lines, arcs, surfaces, cubes and cylinders. While generating process plan it is necessary to convert the CAD data into design oriented geometric entities, into manufacturing related features such as plane cylinders, taper cylinders, holes, slots, pockets. This process is tedious and time taking. Ideal CAD/CAM integration requires machining processes and sequence of operations should be generated automatically. In the research work, automatic process plan module convert the design data from the modeling module into manufacturing information using the knowledge based database. The entire flow of process in terms of algorithms is explained in Figure 6.

The CAPP system developed is composed of different activities.

1. Machining features generated in the design stage are recognized and stored under the Visual Basic control of CATIA.

Software Active X interface.

2. The feature list recognized and stored is utilized to develop the process plan by linking with main source file. Based on the part body recognized from the feature list, respective knowledge base is used for the respective features such as plane cylinder, through hole and blind hole.

3. Determination of the machining operations for the identified features and considers the constraints associated with the dimensions.

4. Determination of the machining sequence for fixturing the part according to its number of set ups.

5. Determination of the cutting tools considering the machine-tool, dimensions and tool geometry.

6. Determination of the cutting conditions considering the tool parameters, machining features material, machine capacity.

7. Then the process planning has been generated as shown in Table 1. 


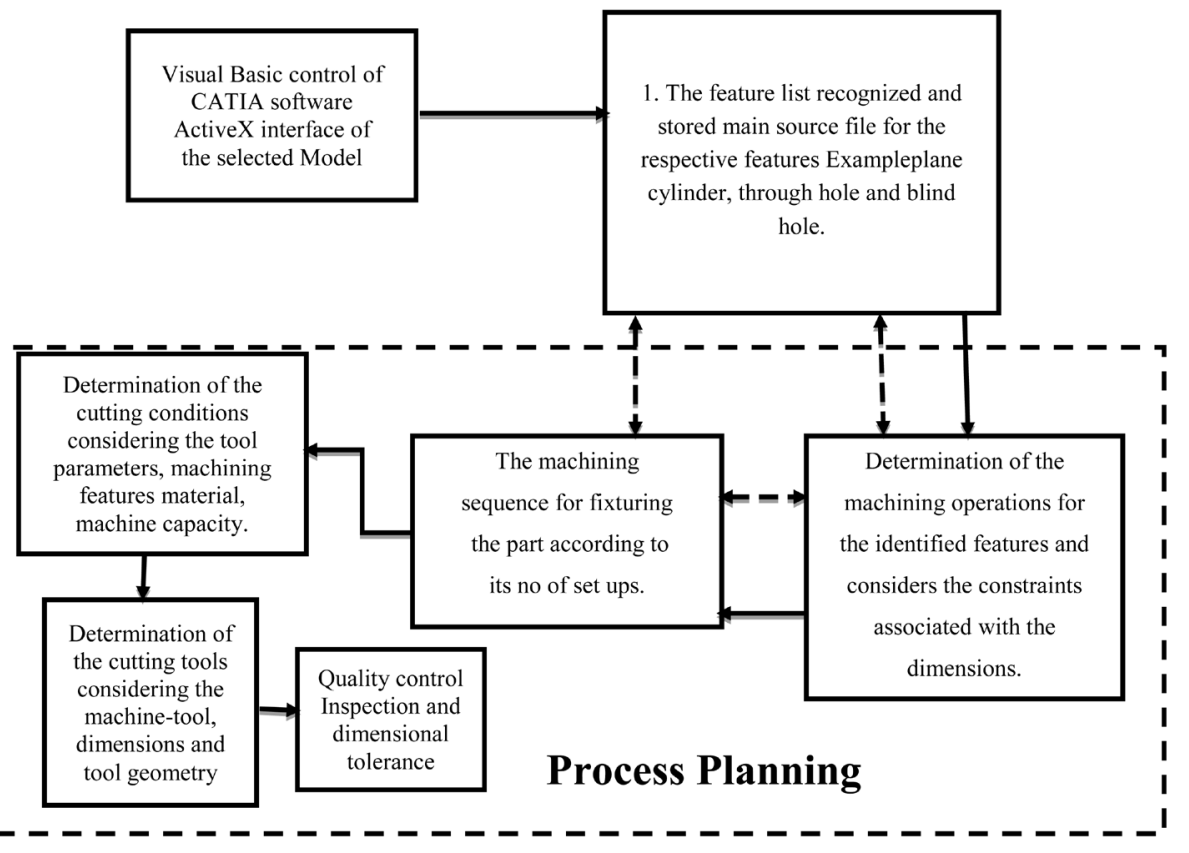

Figure 6. Flow chart for proposed methodology.

Table1. Process planning.

\begin{tabular}{|c|c|c|c|c|c|}
\hline Operation & Machine & Tool & Z-start & Depth & Speed (m/min) \\
\hline $\begin{array}{c}\text { Integrated } \\
\text { Rough/Finish Milling }\end{array}$ & VMC-400 M & Ball-nose & $0 \%$ to $2 \% \mathrm{~L}$ & $\begin{array}{l}2 \% \text { length in total length of } \\
\text { cylindrical prismatic part [L] }\end{array}$ & 38.48 \\
\hline Center Drill through hole & VMC-400 M & Drill two flute & 0 to $\mathrm{L}$ & $\begin{array}{l}\text { Equal to height length of } \\
\text { cylindrical prismatic part }\end{array}$ & 18.03 \\
\hline Blind Hole & VMC-400 M & Drill one/two flute & 0 to 1 & Equal to the height of blind hole feature & 18.33 \\
\hline
\end{tabular}

\section{Conclusion}

This work attempts to develop algorithms for modeling the components through the input of machining features. As a result, feature information is directly available to downstream activities, and feature extraction is no longer needed. Hence, it is economical in terms of time and cost compared to available feature based techniques. The feature based approach described is capable to perform process plan generation for the milling components based on the input features given in modeling. The system enables a reduction of overall lead time and improves the efficiency of the machined components. Machining features generated in the design stage are recognized and stored under the Visual Basic control of CATIA software ActiveX interface. Algorithms are developed for individual features and these algorithms are embedded in Visual Basic forms. This system was discussed for 2.5 Dimensional part approach and best suited for 2.5D parts. However, that can be extended to 3 dimensional prismatic part and complex features machining. Finally a process planning chart has been presented as a model process planning. In the present study takes up only few machining features, and can be extended for more features to arrive at a complete process plan for any real time component. This approach saves the time of machining and easy understanding of process planning to everyone. On the other hand the total cost of manufacturing a product can be reduced without compromising in its specified quality.

\section{References}

[1] Dickinson, S.J. and Zucker, S.W. (2009) Shock Graphs and Shape Matching. International Journal of Computer Vision, 5, 10-13.

[2] Zhou, J. (2008) FSMT: A Feature Solid-Modelling Tool for Feature-Based Design and Manufacture. Computer Aided Design, 3, 2-12. 
[3] Lou, K., Prabhakar, S. and Ramani, S. (2004) Content Based Three Dimensional Engineering Shape Search. Proceedings of the 20th International Conference on Data Engineering, Washington DC, 30 March-2 April 2004, 754-765. http://dx.doi.org/10.1109/ICDE.2004.1320043

[4] Mantyla, M. (2007) Feature Modelling by Incremental Feature Recognition. Computer Aided Design, 5, 47-59.

[5] Iyer, N., Lou, K., Jayanti, S., Kalyanaraman, Y. and Ramani, K. (2004) A Multi-Scale Hierarchical 3D Shape Representation for Similar Shape Retrieval. Tools and Methods of Competitive Engineering Conference, Lausanne, 13-17 April 2004, 1117-1128.

[6] Ramesh, M.M., Hoi, D.Y. and Dutta, D. (2001) Feature-Based Shape Similarity Measurement for Retrieval of Mechanical Parts. Journal of Computing and Information Science in Engineering, 1, 245-256. http://dx.doi.org/10.1115/1.1412456

[7] Ovtcharova, J. (2009) Feature-Based Modelling by Integrating Design and Recognition Approaches. Computer Aided Design, 2, 46-65.

[8] Roerdink, J.B. (2002) Similarity Measures for Convex Polyhedra Based on Minkowski Addition. Pattern Recognition, 3, 99-110.

[9] Requicha, A.A.G. (2007) Integration of Feature Based Design and Feature Recognition. Computer Aided Design, 9, 93-104.

[10] Shokoufandeh, A. (2003) Reeb Graph Based Shape Retrieval for CAD. Proceedings of 23rd ASME DETC Computers and Information in Engineering (CIE) Conference, Chicago, 2-6 September 2003, DETC2003/CIE-48215.

[11] Kim, K. (2008) A Feature-Based Approach to Extracting Machining Features. Computer Aided Design, 3, $101-135$.

[12] Karnik, M.V., Gupta, S.K. and Magrab, E.B. (2005) Geometric Algorithms for Containment Analysis of Rotational Parts. Computer Aided Design, 37, 213-230. http://dx.doi.org/10.1016/j.cad.2004.06.007

[13] Cicirello, V. and Regli, W.C. (2012) An Approach to Feature Based Comparison of Solid Models of Machined Parts. Journal of Artificial Intelligence for Engineering Design Analysis and Manufacturing, 6, 385-399.

[14] Devireddy, C.R. and Ghosh, K. (2010) Feature-Based Modeling and Neural Networks-Based CAPP for Integrated Manufacturing. International Journal of Computer Integrated Manufacturing, 2, 51-59.

[15] Abu Bakar, N. and Juri, A.H. (2008) Recognition of Cylindrical Based Features Using Edge Boundary Technique for Integrated Manufacturing. Robotics and Computer Integrated Manufacturing, 10, 41-62.

[16] Pande, S.S. (2010) An Intelligent Feature-Based Process Planning System for Prismatic Parts. International Journal of Production Research, 4, 431-447. 
Scientific Research Publishing (SCIRP) is one of the largest Open Access journal publishers. It is currently publishing more than 200 open access, online, peer-reviewed journals covering a wide range of academic disciplines. SCIRP serves the worldwide academic communities and contributes to the progress and application of science with its publication.

Other selected journals from SCIRP are listed as below. Submit your manuscript to us via either submit@scirp.org or Online Submission Portal.
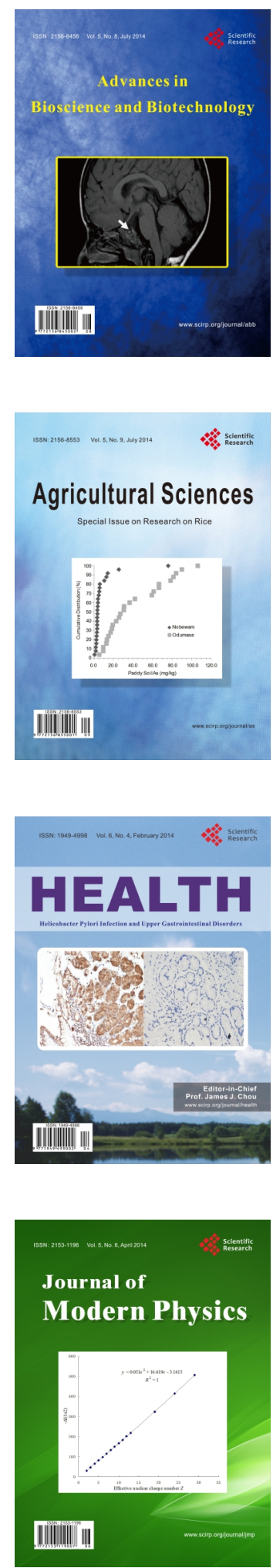
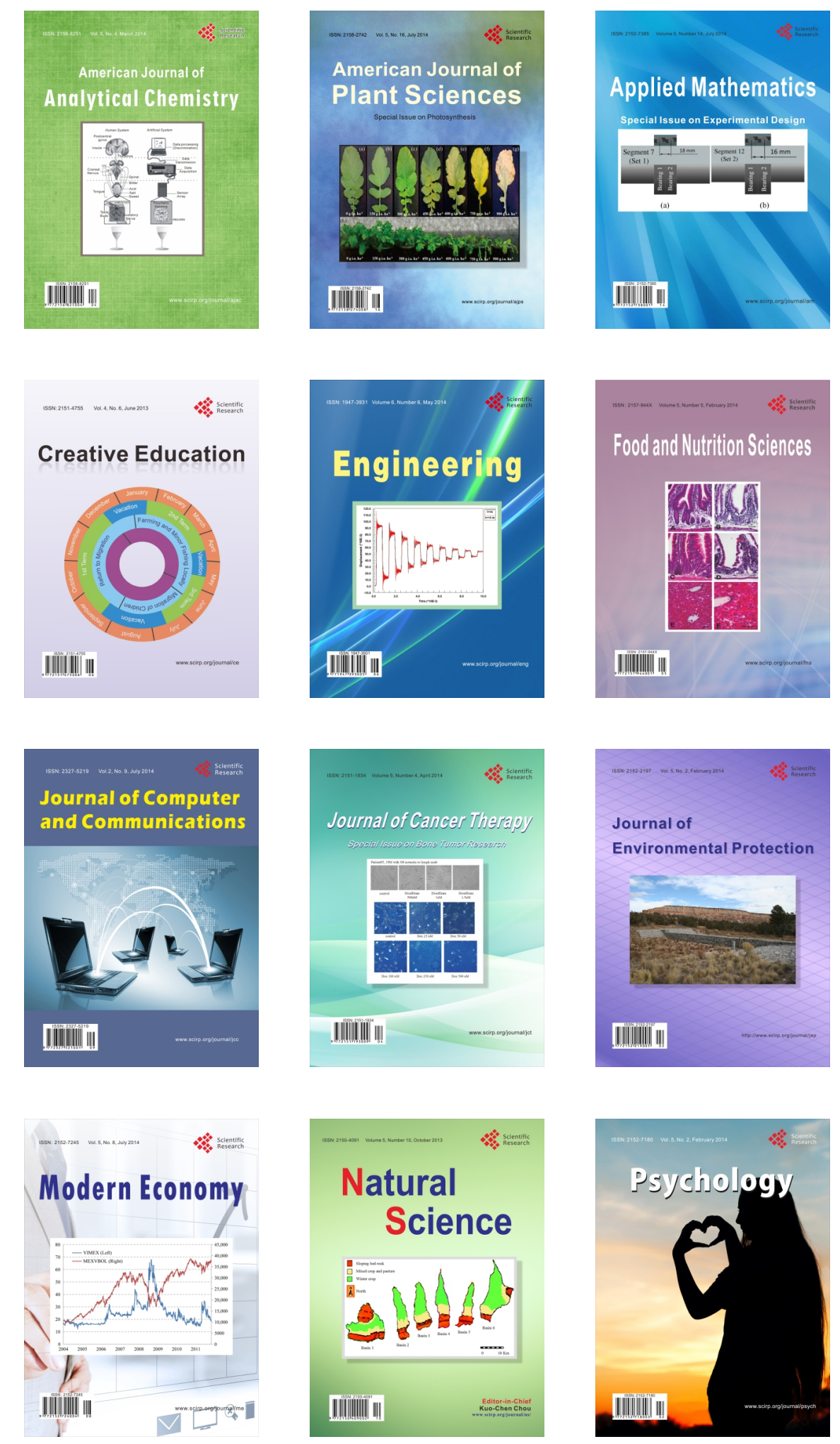